\title{
Structure of stable degeneration of K3 surfaces into pairs of rational elliptic surfaces
}

\author{
Yusuke Kimura \\ KEK Theory Center, Institute of Particle and Nuclear Studies, KEK, \\ 1-1 Oho, Tsukuba, Ibaraki 305-0801, Japan \\ E-mail: kimurayu@post.kek.jp
}

ABSTRACT: F-theory/heterotic duality is formulated in the stable degeneration limit of a K3 fibration on the F-theory side. In this note, we analyze the structure of the stable degeneration limit. We discuss whether stable degeneration exists for pairs of rational elliptic surfaces. We demonstrate that, when two rational elliptic surfaces have an identical complex structure, stable degeneration always exists. We provide an equation that systematically describes the stable degeneration of a K3 surface into a pair of isomorphic rational elliptic surfaces. When two rational elliptic surfaces have different complex structures, whether their sum glued along a smooth fiber admits deformation to a K3 surface can be determined by studying the structure of the K3 lattice. We investigate the lattice theoretic condition to determine whether a deformation to a K3 surface exists for pairs of extremal rational elliptic surfaces. In addition, we discuss the configurations of singular fibers under stable degeneration.

The sum of two isomorphic rational elliptic surfaces glued together admits a deformation to a K3 surface, the singular fibers of which are twice that of the rational elliptic surface. For special situations, singular fibers of the resulting K3 surface collide and they are enhanced to a fiber of another type. Some K3 surfaces become attractive in these situations. We determine the complex structures and the Weierstrass forms of these attractive K3 surfaces. We also deduce the gauge groups in F-theory compactifications on these attractive $\mathrm{K} 3$ surfaces times a K3. $E_{6}, E_{7}, E_{8}, \mathrm{SU}(5)$, and $\mathrm{SO}(10)$ gauge groups arise in these compactifications.

KEywords: Differential and Algebraic Geometry, F-Theory, Gauge Symmetry, Superstring Vacua

ArXiv EPrint: 1710.04984 


\section{Contents}

1 Introduction 1

2 Stable degeneration of $\mathrm{K3}$ to a pair of isomorphic rational elliptic surfaces 3

2.1 Equation for the degeneration of $\mathrm{K} 3$ to a pair of isomorphic rational elliptic surfaces 3

Extremal rational elliptic surfaces

2.3 Example of generic deformation using an extremal rational elliptic surface

2.4 Attractive K3 surfaces as a special deformation of two extremal rational elliptic surfaces glued together

2.4.1 Complex structures of attractive K3 surfaces, and gauge groups in F-theory compactifications

2.4.2 Weierstrass equation

2.4.3 Anomaly cancellation condition

3 Stable degeneration of K3 to a pair of non-isomorphic rational elliptic surfaces

$\begin{array}{lll}3.1 & \text { Lattice condition for stable degeneration limit } & 14\end{array}$

$\begin{array}{lll}3.2 & \text { Pairs of extremal rational elliptic surfaces } & 15\end{array}$

\section{Introduction}

The F-theory approach to particle physics model building has several advantages. It naturally realizes $\mathrm{SU}(5)$ grand unified theories with matter in spinor representations of $\mathrm{SO}(10)$. In contrast to D-brane models, there is no difficulty in generating up-type Yukawa couplings. Furthermore, it can evade the problem of weakly coupled heterotic string theory addressed in [1]. Recent studies on F-theory model building [2-5] have emphasized the use of local models. However, in order to address the issue of gravity such as inflation, a global model of compactification need to be considered eventually. In particular, many insights can be gained by the duality between heterotic string and F-theory [6-10], which states the equivalence between the former compactified on an elliptically fibered CY $n$-fold and the latter on a K3 fibered CY $(n+1)$-fold in the stable degeneration limit $[10,11] .^{1}$ The aim of the present paper is to develop a systematic study of the process of stable degeneration of a K3 surface for a pair of rational elliptic surfaces.

\footnotetext{
${ }^{1}$ For recent discussion of the stable degeneration limit of F-theory and F-theory/heterotic duality, see, for example, [12-16].
} 
K3 surface stably degenerates into two rational elliptic surfaces in two distinct ways:

i) K3 surface splits into two rational elliptic surfaces with an identical complex structure

ii) K3 surface splits into two rational elliptic surfaces with different complex structures.

We discuss these cases separately, in section 2 and section 3, respectively.

We demonstrate in section 2.1 that, when a pair of rational elliptic surfaces are isomorphic, stable degeneration can be described by a systematic equation. We analyze the geometry of stable degeneration of the first kind i) using this equation. We determine that given any pair of isomorphic rational elliptic surfaces, there is some K3 surface that stably degenerates into the pair.

However, it is considerably difficult to describe stable degeneration by an equation when two rational elliptic surfaces have different complex structures. Moreover, a pair of non-isomorphic rational elliptic surfaces glued together along a smooth fiber does not necessarily admit a deformation to a K3 surface. Complex structures and configurations of singular fibers are classified for a specific class of rational elliptic surfaces, called extremal rational elliptic surfaces. We focus on the extremal rational elliptic surfaces to analyze the stable degeneration of the second kind ii). We determine whether stable degeneration exists for pairs of these rational elliptic surfaces. We use the lattice theoretic approach to analyze this process.

We also study the configuration of singular fibers under the stable degeneration limit. In F-theory, non-Abelian gauge symmetries on the 7-branes are in correspondence with the types of singular fibers. Therefore, analyzing the configurations of singular fibers under the stable degeneration limit is of physical interest.

The outline of this study is as follows. In section 2, we discuss the stable degeneration limit where a K3 surface degenerates into two isomorphic rational elliptic surfaces. We provide an equation that systematically describes this process. We determine that any pair of isomorphic rational elliptic surfaces glued together deforms to a K3 surface, which is a double cover of $\mathbb{P}^{2}$ ramified over a sextic curve. Moreover, we discuss the configurations of singular fibers under the degeneration. Furthermore, we review the properties of the extremal rational elliptic surfaces. We discuss some examples of stable degeneration using an extremal rational elliptic surface. The sum of two isomorphic rational elliptic surfaces glued together admits a deformation to a K3 surface, the singular fibers of which are twice that of the rational elliptic surface. For special situations, two fibers of the same type of the resulting K3 surface collide, and they are enhanced to a fiber of another type. These situations can be considered as special cases of stable degeneration. For such situations, some K3 surfaces become attractive. In section 2.4, we determine the complex structures of these attractive K3 surfaces and their Weierstrass forms. We also deduce the nonAbelian gauge symmetries that form on the 7-branes in F-theory compactifications on these attractive $\mathrm{K} 3$ surfaces times a $\mathrm{K} 3 . E_{6}, E_{7}, E_{8}, \mathrm{SU}(5)$, and $\mathrm{SO}(10)$ gauge groups arise in these models. In section 3, we investigate the stable degeneration limit where a K3 surface degenerates into two non-isomorphic rational elliptic surfaces. Whether such a degeneration exists can be determined by studying the lattice structure of the second integral cohomology 
group $H^{2}(S, \mathbb{Z})$ of a $\mathrm{K} 3$ surface $S$. We obtain the lattice condition under which two nonisomorphic rational elliptic surfaces glued together admits a deformation to a K3 surface. We determine whether a deformation to a K3 surface exists for pairs of extremal rational elliptic surfaces. We study the configuration of singular fibers under stable degeneration using the lattice theoretic argument. We state the concluding remarks in section 4 .

\section{Stable degeneration of $\mathrm{K} 3$ to a pair of isomorphic rational elliptic surfaces}

\subsection{Equation for the degeneration of K3 to a pair of isomorphic rational el- liptic surfaces}

In this section, we discuss the stable degeneration of a $\mathrm{K} 3$ surface to two isomorphic rational elliptic surfaces. We provide an equation that describes such stable degeneration systematically. From this equation, we determine that any pair of isomorphic rational elliptic surfaces admits stable degeneration (i.e., there is a K3 surface that splits into that pair of rational elliptic surfaces in the stable degeneration limit). We also conclude that there always exists some appropriate K3 surface whose singular fibers are the sum of the singular fibers of the two isomorphic rational elliptic surfaces to which K3 surface degenerates.

When we discuss a rational elliptic surface in this study, we only consider such a surface with a section. The base space of a rational elliptic surface is isomorphic to $\mathbb{P}^{1}$ by Lüroth's theorem, and it is known that every rational elliptic surface with a section is the blow-up of $\mathbb{P}^{2}$ in the nine base points of a cubic pencil [17]. The Picard number of a rational elliptic surface is 10, and the rank of the Mordell-Weil group ranges from 0 to 8 :

$$
0 \leq \mathrm{rkMW} \leq 8
$$

A generic rational elliptic surface has Mordell-Weil rank 8. Rational elliptic surfaces with Mordell-Weil rank 0 are called extremal rational elliptic surfaces. For extremal rational elliptic surfaces, the rank of the singularity type is 8 , which is the highest for a rational elliptic surface. We will review the properties of extremal rational elliptic surfaces in section 2.2. In section 3, we focus on extremal rational elliptic surfaces to discuss the stable degeneration of a K3 surface into a pair of non-isomorphic rational elliptic surfaces.

When two rational elliptic surfaces have isomorphic smooth elliptic fibers, we can glue two rational elliptic surfaces in the following fashion: we choose a point in base $\mathbb{P}^{1}$ of each rational elliptic surface over which the fiber is smooth and isomorphic, and we glue two rational elliptic surfaces by identifying the isomorphic smooth fibers over the chosen points. As described in section 3, when a certain lattice theoretic condition is satisfied, the sum of two (not necessarily isomorphic) rational elliptic surfaces glued together can be deformed to a K3 surface.

In this section, we particularly consider the case wherein two rational elliptic surfaces that are glued together are isomorphic. For this particular case, we explicitly provide an equation that describes the stable degeneration of a K3 surface into the pair of isomorphic 
rational elliptic surfaces glued together. As stated above, a rational elliptic surface $X$ is the blow-up of $\mathbb{P}^{2}$ in the base points of a cubic pencil; we denote the cubic pencil of a rational elliptic surface $X$ as $f$. The double cover of $\mathbb{P}^{2}$ ramified along a degree 6 curve is, in general, a $\mathrm{K} 3$ surface. We particularly consider double covers of $\mathbb{P}^{2}$ ramified over a degree 6 curve given by the following form of equations:

$$
\tau^{2}=f g,
$$

where $g$ is a polynomial of degree 3 and $g$ is the cubic pencil of the same type as the pencil $f$, but the ratio of the coefficients of the pencil $g$ is generally different from the ratio of the coefficients of the pencil $f$. For this situation, K3 surface (2.2) is elliptically fibered. To be explicit, when the cubic pencil $f$ is given by

$$
f=a h_{1}+b h_{2},
$$

where $a, b$ are coefficients of the pencil $f$, we choose the cubic pencil $g$ as follows:

$$
g=c h_{1}+d h_{2} \text {. }
$$

$c, d$ are coefficients of the pencil $g$, and the ratio $[c: d]$ is generally different from the ratio $[a: b]$. For the limit at which polynomial $g$ goes to cubic pencil $f$ (i.e. for the limit at which ratio $[c: d]$ goes to the ratio $[a: b]$ ), equation $(2.2)$ is split into the following two equations:

$$
\begin{aligned}
\tau & =f \\
\tau & =-f .
\end{aligned}
$$

Each of the above two equations in (2.5) describes a rational elliptic surface given by cubic pencil $f$; therefore, when a cubic polynomial $g$ goes to cubic pencil $f$, the K3 surface $(2.2)$ splits into two isomorphic rational elliptic surfaces, each given by the cubic pencil $f$. This is the stable degeneration limit of the K3 surface (2.2) splitting into two copies of rational elliptic surfaces $X$. Thus, we conclude that two isomorphic rational elliptic surfaces glued along an isomorphic smooth fiber always admit a deformation to a K3 surface.

We only consider rational elliptic surfaces with a global section in this note; therefore, they admit transformation into the Weierstrass form. The coefficients of the Weierstrass form depend on the coordinate of the base $\mathbb{P}^{1}$. We denote the homogeneous coordinate of the base $\mathbb{P}^{1}$ as $[u: v]$. In terms of the coordinate $[u: v]$, the stable degeneration of a K3 surface (2.2) splitting into two isomorphic rational elliptic surfaces is described by the following equation:

$$
\tau^{2}=u^{2}+2 k u v+v^{2} .
$$

$k$ in equation (2.6) denotes a parameter of deformation. $k$ varies along the deformation, and when $k$ assumes the values

$$
k= \pm 1 \text {, }
$$

equation (2.6) splits into linear factors. This occurs when a K3 surface splits into two isomorphic rational elliptic surfaces. 
We observe from equation (2.6) that the K3 surface (2.2) is the quadratic base change of the rational elliptic surface (2.5) into which the K3 surface splits in the stable degeneration limit, when $g$ is the cubic pencil of the same type as the pencil $f$. In other words, the Weierstrass equation of the K3 surface is obtained when some appropriate quadratic equations are substituted into variables $u, v$ in the coefficients of the Weierstrass form of a rational elliptic surface. Thus, the generic K3 surface (2.2) that results from the deformation of two isomorphic rational elliptic surfaces (2.5) glued together has twice the number of singular fibers as a rational elliptic surface.

For special situations, singular fibers of the same type of the K3 surface, that is obtained as the quadratic base change of a rational elliptic surface, collide and they are enhanced to a fiber of another type. We discuss these situations in section 2.4.

The aforementioned argument applies to the deformation of every pair of two isomorphic rational elliptic surfaces with a global section glued along smooth fiber to a K3 surface.

\subsection{Extremal rational elliptic surfaces}

We summarize the properties of extremal rational elliptic surfaces. In section 2.3, we discuss the quadratic base change of extremal rational elliptic surfaces that ramifies only over smooth fibers. In section 2.4, we discuss the limits at which singular fibers of the same type collide in the quadratic base change of extremal rational elliptic surfaces. We discuss the structures of attractive K3 surfaces that result from the quadratic base change of extremal rational elliptic surfaces in section 2.4.

Extremal rational elliptic surfaces have the singularity type of rank 8, and the MordellWeil groups only have torsion parts. Singular fiber types of the extremal rational elliptic surfaces are classified, and the complex structure of an extremal rational elliptic surface is uniquely determined by the fiber type, except for the surfaces with the fiber type $\left[I_{0}^{*}, I_{0}^{*}\right][18]$. J-invariant of fibers of an extremal rational elliptic surface with the fiber type $\left[I_{0}^{*}, I_{0}^{*}\right]$ is constant over the base $\mathbb{P}^{1}$, and the complex structure of a rational elliptic surface with the fiber type $\left[I_{0}^{*}, I_{0}^{*}\right]$ depends on the value of $\mathrm{j}$. The complex structure of an extremal rational elliptic surface with the fiber type $\left[I_{0}^{*}, I_{0}^{*}\right]$ is fixed when the j-invariant of the fiber is chosen.

Provided these facts, in this note, we denote an extremal rational elliptic surface using its fiber type as the subscript. For example, an extremal rational elliptic surface with the fiber type $\left[I V, I V^{*}\right]$ is denoted as $X_{\left[I V, I V^{*}\right]}$. We simply use $n$ to represent the $I_{n}$ fiber, and $m^{*}$ to represent the $I_{m}^{*}$ fiber. Therefore, an extremal rational elliptic surface with singular fibers of type $I V^{*}, I_{3}$, and $I_{1}$ is denoted as $X_{\left[I V^{*}, 3,1\right]}$. We denote a surface with the fiber type $\left[I_{0}^{*}, I_{0}^{*}\right]$ as $X_{\left[0^{*}, 0^{*}\right]}(j)$, because the complex structure of such a surface depends on the j-invariant of fibers. We list the configurations of singular fiber types of the extremal rational elliptic surfaces in table 1 . The Weierstrass forms of the extremal rational elliptic surfaces were also derived in [18]. We include the Weierstrass forms of the extremal rational elliptic surfaces in table 1.

The cubic pencils for all the extremal rational elliptic surfaces, except the surfaces $X_{\left[I I, I I^{*}\right]}, X_{\left[I I I, I I I^{*}\right]}, X_{\left[I V, I V^{*}\right]}, X_{\left[0^{*}, 0^{*}\right]}(j)$, were obtained in [19]. The cubic pencil for the 


\begin{tabular}{|c|c|c|c|}
\hline $\begin{array}{l}\text { Extremal rational } \\
\text { elliptic surface }\end{array}$ & Fiber type & $a_{4}$ & $a_{6}$ \\
\hline$X_{\left[I I, I I^{*}\right]}$ & $I I^{*}, I I$ & 0 & $u v^{5}$ \\
\hline$X_{\left[I I I, I I I^{*}\right]}$ & $I I I^{*}, I I I$ & $u v^{3}$ & 0 \\
\hline$X_{\left[I V, I V^{*}\right]}$ & $I V^{*}, I V$ & 0 & $u^{2} v^{4}$ \\
\hline$X_{\left[0^{*}, 0^{*}\right]}(j)$ & $I_{0}^{*}, I_{0}^{*}$ & $s u^{2} v^{2}$ & $t u^{3} v^{3}$ \\
\hline$X_{\left[I I^{*}, 1,1\right]}$ & $I I^{*} I_{1} I_{1}$ & $-3 u^{4}$ & $2 u^{5} v$ \\
\hline$X_{\left[I I I^{*}, 2,1\right]}$ & $I I I^{*} I_{2} I_{1}$ & $-u v^{3}$ & $v^{5}(u-v)$ \\
\hline$X_{\left[I V^{*}, 3,1\right]}$ & $I V^{*} I_{3} I_{1}$ & $v^{3}(24 u-27 v)$ & $v^{4}\left(16 u^{2}-72 u v+54 v^{2}\right)$ \\
\hline$X_{\left[4^{*}, 1,1\right]}$ & $I_{4}^{*} I_{1} I_{1}$ & $-3 v^{2}\left(u^{2}-3 v^{2}\right)$ & $u v^{3}\left(2 u^{2}-9 v^{2}\right)$ \\
\hline$X_{\left[2^{*}, 2,2\right]}$ & $I_{2}^{*} I_{2} I_{2}$ & $-3 u v(u-v)^{2}$ & $(u-v)^{3}\left(u^{3}+v^{3}\right)$ \\
\hline$X_{[1 *, 4,1]}$ & $I_{1}^{*} I_{4} I_{1}$ & $-3(u-2 v)^{2}\left(u^{2}-3 v^{2}\right)$ & $u(u-2 v)^{3}\left(2 u^{2}-9 v^{2}\right)$ \\
\hline$X_{[9,1,1,1]}$ & $\begin{array}{llll}I_{9} & I_{1} & I_{1} & I_{1} \\
\end{array}$ & $-3 u\left(u^{3}+24 v^{3}\right)$ & $2\left(u^{6}+36 u^{3} v^{3}+216 v^{6}\right)$ \\
\hline$X_{[8,2,1,1]}$ & $\begin{array}{llll}I_{8} & I_{2} & I_{1} & I_{1} \\
\end{array}$ & $-3\left(u^{4}+4 u^{2} v^{2}+v^{4}\right)$ & $2 u^{6}+12 u^{4} v^{2}+15 u^{2} v^{4}-2 v^{6}$ \\
\hline$X_{[6,3,2,1]}$ & $\begin{array}{llll}I_{6} & I_{3} & I_{2} & I_{1}\end{array}$ & $-3\left(u^{4}+4 u^{3} v-2 u v^{3}+v^{4}\right)$ & $\begin{array}{c}2 u^{6}+12 u^{5} v+12 u^{4} v^{2}-14 u^{3} v^{3} \\
+3 u^{2} v^{4}-6 u v^{5}+2 v^{6}\end{array}$ \\
\hline$X_{[5,5,1,1]}$ & $\begin{array}{lllll}I_{5} & I_{5} & I_{1} & I_{1}\end{array}$ & $\begin{array}{c}-3\left(u^{4}-12 u^{3} v+14 u^{2} v^{2}\right. \\
\left.+12 u v^{3}+v^{4}\right)\end{array}$ & $\begin{array}{l}2\left(u^{6}-18 u^{5} v+75 u^{4} v^{2}\right. \\
\left.+75 u^{2} v^{4}+18 u v^{5}+v^{6}\right)\end{array}$ \\
\hline$X_{[4,4,2,2]}$ & $\begin{array}{llll}I_{4} & I_{4} & I_{2} & I_{2} \\
\end{array}$ & $-3\left(u^{4}-u^{2} v^{2}+v^{4}\right)$ & $2 u^{6}-3 u^{4} v^{2}-3 u^{2} v^{4}+2 v^{6}$ \\
\hline$X_{[3,3,3,3]}$ & $\begin{array}{llll}I_{3} & I_{3} & I_{3} & I_{3} \\
\end{array}$ & $-3\left(u^{4}-8 u v^{3}\right)$ & $2\left(u^{6}+20 u^{3} v^{3}-8 v^{6}\right)$ \\
\hline
\end{tabular}

Table 1. Fiber types of the extremal rational elliptic surfaces, and coefficients $a_{4}, a_{6}$ of the Weierstrass form $y^{2}=x^{3}+a_{4} x+a_{6}$. $[u: v]$ is the homogeneous coordinate on the base $\mathbb{P}^{1}$. For surface $X_{\left[0^{*}, 0^{*}\right]}(j), s, t$ in coefficients $a_{4}, a_{6}$ of the Weierstrass form are complex numbers, $s, t \in \mathbb{C}$, with $4 s^{3}+27 t^{2} \neq 0 . j$-invariant of fibers of surface $X_{\left[0^{*}, 0^{*}\right]}(j)$ is a function of $s, t$.

extremal rational elliptic surface $X_{\left[I V, I V^{*}\right]}$ is given by

$$
a y z(y+z)+b x^{3}
$$

$[x: y: z]$ represents the homogeneous coordinates on $\mathbb{P}^{2}$, and $[a: b]$ represents the homogeneous coordinate on $\mathbb{P}^{1}$. The cubic pencils of extremal rational elliptic surfaces are listed in table 2 .

\subsection{Example of generic deformation using an extremal rational elliptic surface}

We discuss an example of results in section 2.1 using an extremal rational elliptic surface. We consider the surface $X_{\left[I V, I V^{*}\right]}$. As stated in section 2.2, the cubic pencil of surface $X_{\left[I V, I V^{*}\right]}$ is given by

$$
f=a y z(y+z)+b x^{3} .
$$




\begin{tabular}{|c|c|}
\hline Extremal rational elliptic surface & Pencil \\
\hline$X_{\left[I I^{*}, 1,1\right]}$ & $a\left\{3\left(x^{2}-y^{2}\right) z+2 x^{3}\right\}+b z^{3}$ \\
\hline$X_{\left[I I I^{*}, 2,1\right]}$ & $a z\left\{y^{2}+x(z-2 x)\right\}+b x^{3}$ \\
\hline$X_{\left[I V^{*}, 3,1\right]}$ & $a x y z+b(x+y+z)^{3}$ \\
\hline$X_{\left[I V, I V^{*}\right]}$ & $a y z(y+z)+b x^{3}$ \\
\hline$X_{\left[4^{*}, 1,1\right]}$ & $a\left\{(2 x+z)\left(x z+y^{2}\right)+x^{3}\right\}+b x\left(x z+y^{2}\right)$ \\
\hline$X_{\left[2^{*}, 2,2\right]}$ & $a(x+y)\left(x y+z^{2}\right)+b x^{2} y$ \\
\hline$X_{\left[1^{*}, 4,1\right]}$ & $a x\left\{(x+y) z+y^{2}\right\}+b(x+y) z^{2}$ \\
\hline$X_{[9,1,1,1]}$ & $a\left(x^{2} y+y^{2} z+z^{2} x-3 x y z\right)+b x y z$ \\
\hline$X_{[8,2,1,1]}$ & $a\left\{x\left(x z-y^{2}\right)+y^{2} z\right\}+b(y+2 z)\left(x z-y^{2}+z^{2}\right)$ \\
\hline$X_{[6,3,2,1]}$ & $a z\left(x^{2}+x y+x z+y^{2}\right)+b\left(-x^{2} z+x y z+y^{3}\right)$ \\
\hline$X_{[5,5,1,1]}$ & $a y z(x+y+z)+b x(x+y)(x+z)$ \\
\hline$X_{[4,4,2,2]}$ & $a(x-y)\left(x y-z^{2}\right)+b x y(x+y-2 z)$ \\
\hline$X_{[3,3,3,3]}$ & $a\left(x^{3}+y^{3}+z^{3}\right)+b x y z$ \\
\hline
\end{tabular}

Table 2. Pencils of extremal rational elliptic surfaces.

We fix $a, b$ to be non-zero constants in the cubic pencil $f$. We consider the cubic polynomial $g$ to be the pencil of the same type as the cubic pencil $f$ :

$$
g=c y z(y+z)+d x^{3} .
$$

We fix $c, d$ to be non-zero constants. We choose $c, d$ so that the ratio $[c: d]$ is generally different from the ratio $[a: b]$ of the coefficients $a, b$ in the pencil $f$. For the cubic pencil $f(2.9)$ and cubic pencil $g(2.10)$, equation

$$
\tau^{2}=f g
$$

generically provides a K3 surface with two type $I V^{*}$ fibers and two type $I V$ fibers. For the limit at which ratio $[c: d]$ goes to $[a: b]$, the cubic pencil $g$ coincides with the cubic pencil $f$. In this stable degeneration limit, equation (2.11) is split into linear factors; accordingly, the K3 surface (2.11) splits into two extremal rational elliptic surfaces $X_{\left[I V, I V^{*}\right]}$.

We stated in section 2.1 that gluing together two isomorphic rational elliptic surfaces, each given by cubic pencil $f$, to obtain a K3 surface (2.2) is equivalent to the quadratic base change of a rational elliptic surface over $\mathbb{P}^{1}$, when the cubic pencil $g$ is chosen to be the same type as the pencil $f$. We consider, as an example, the case wherein two extremal rational elliptic surfaces $X_{\left[I V, I V^{*}\right]}$ are glued together to form a K3 surface, to demonstrate this explicitly. We discuss the generic situation in which the quadratic base change ramifies 
over smooth fibers. As given in table 1, the Weierstrass form of $X_{\left[I V, I V^{*}\right]}$ is given by

$$
y^{2}=x^{3}+u^{2} v^{4}
$$

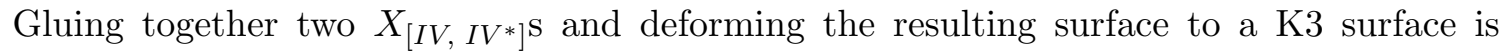
equivalent to substituting the following quadratic equations into variables $u, v$ :

$$
\begin{aligned}
& u=\alpha_{1} \tilde{u}^{2}+\alpha_{2} \tilde{u} \tilde{v}+\alpha_{3} \tilde{v}^{2}=\alpha_{1}\left(\tilde{u}-\beta_{1} \tilde{v}\right)\left(\tilde{u}-\beta_{2} \tilde{v}\right) \\
& v=\alpha_{4} \tilde{u}^{2}+\alpha_{5} \tilde{u} \tilde{v}+\alpha_{6} \tilde{v}^{2}=\alpha_{4}\left(\tilde{u}-\beta_{3} \tilde{v}\right)\left(\tilde{u}-\beta_{4} \tilde{v}\right) .
\end{aligned}
$$

$\alpha_{i}, i=1, \cdots, 6$, are some constants, and the quadratic terms are split into linear factors on the right extreme hand sides in equation (2.13). (In (2.13), we assume that $\beta_{1} \neq \beta_{2}$ and $\beta_{3} \neq \beta_{4}$.) The resulting K3 surface has the following Weierstrass form:

$$
y^{2}=x^{3}+\alpha_{1}^{2} \alpha_{4}^{4}\left(\tilde{u}-\beta_{1} \tilde{v}\right)^{2}\left(\tilde{u}-\beta_{2} \tilde{v}\right)^{2}\left(\tilde{u}-\beta_{3} \tilde{v}\right)^{4}\left(\tilde{u}-\beta_{4} \tilde{v}\right)^{4} .
$$

We confirm from equation (2.14) that the resulting K3 surface has two type $I V^{*}$ fibers and two type $I V$ fibers, for generic constants $\alpha_{i}, i=1, \cdots, 6$.

When $\beta_{1}=\beta_{2}$, two fibers of type $I V$ collide and they are enhanced to a type $I V^{*}$ fiber. For this special case, we obtain a K3 surface with three $I V^{*}$ fibers. Situations of this kind, in which singular fibers of the same type collide in the quadratic base change of an extremal rational elliptic surface, will be discussed in section 2.4.

\subsection{Attractive K3 surfaces as a special deformation of two extremal rational elliptic surfaces glued together}

\subsubsection{Complex structures of attractive K3 surfaces, and gauge groups in F- theory compactifications}

In section 2.1, we mainly discussed the quadratic base change whose ramification occurs over smooth fibers. A smooth fiber remains smooth after the quadratic base change. When the quadratic base change is unramified over a singular fiber, we obtain two copies of that fiber.

As we saw in section 2.1, the quadratic base change of a rational elliptic surface to obtain a K3 surface is the reverse of a process in which a $\mathrm{K} 3$ surface splits into two isomorphic rational elliptic surfaces under stable degeneration. A quadratic base change generically ramifies only over smooth fibers; we obtain two copies of the singular fiber for each singular fiber after the quadratic base change. Thus, the resulting K3 surface has twice as many singular fibers as the rational elliptic surface for such a generic situation. We consider the special situation of the quadratic base change, where singular fibers of the same type collide and they are enhanced to a singular fiber of another type. We particularly consider the examples in which the resulting K3 surfaces after the quadratic base change are enhanced to attractive K3 surfaces. ${ }^{2}$ We show in table 3 the resulting fiber type after the collision of two singular fibers of the same type [20].

\footnotetext{
${ }^{2}$ Following the convention of the term in [21], we refer to a K3 surface with the Picard number 20 as an attractive K3 surface in this study.
} 


\begin{tabular}{|c|c|}
\hline $\begin{array}{c}\text { Original fiber type of } \\
\text { a pair of identical singular fibers }\end{array}$ & Resulting fiber type \\
\hline$I_{n}$ & $I_{2 n}$ \\
$I I$ & $I V$ \\
$I I I$ & $I_{0}^{*}$ \\
$I V$ & $I V^{*}$ \\
\hline
\end{tabular}

Table 3. Resulting fibers after the collision of a pair of singular fibers of the same type.

We consider stable degeneration in which two isomorphic extremal rational elliptic surfaces $X_{\left[I I, I I^{*}\right]}$ are glued together. When two type $I I$ fibers collide in the quadratic base change, we find from table 3 that the resulting fiber has type $I V$. The resulting K3 surface has singular fibers of types $I I^{*}, I I^{*}$, and $I V$. The corresponding $A D E$ type is $E_{8}^{2} \oplus A_{2}$. This is an extremal K3 surface. An extremal K3 surface is an attractive elliptic K3 surface with a global section, with the Mordell-Weil group of rank 0. The extremal K3 surface with $A D E$ type $E_{8}^{2} \oplus A_{2}$ is discussed in [22]. We next consider the gluing of two isomorphic extremal rational elliptic surfaces $X_{\left[I I I, I I I^{*}\right]}$, in which two type $I I I$ fibers collide. The resulting $\mathrm{K} 3$ surface has type $I I I^{*}, I I I^{*}$, and $I_{0}^{*}$ fibers. The corresponding $A D E$ type is $E_{7}^{2} \oplus D_{4}$. This is also an extremal K3 surface. This K3 surface is discussed in [23] as the Jacobian fibration of some K3 genus-one fibration without a section. For the gluing of two isomorphic extremal rational elliptic surfaces $X_{\left[I V, I V^{*}\right]}$, when two type $I V$ fibers collide, the resulting fiber has type $I V^{*}$. Therefore, the resulting K3 surface has three type $I V^{*}$ singular fibers. The corresponding $A D E$ type is $E_{6}^{3}$. This is an extremal K3 surface, and this K3 surface is discussed in [24] as the Jacobian fibration of an attractive K3 genus-one fibration without a section.

For the gluing of two isomorphic extremal rational elliptic surfaces $X_{\left[I I^{*}, 1,1\right]}$, we consider the situation in which two pairs of type $I_{1}$ fibers collide in the quadratic base change. The resulting fibers have type $I_{2}$; the resulting K3 surface has two singular fibers of type $I I^{*}$, and two fibers of type $I_{2}$. When we consider the limit of the quadratic base change of extremal rational elliptic surface $X_{\left[I I I^{*}, 2,1\right]}$ at which two type $I_{2}$ fibers and two type $I_{1}$ fibers collide, the resulting K3 surface has fibers of types $I I I^{*}, I I I^{*}, I_{4}$, and $I_{2}$. The limit of quadratic base change of extremal rational elliptic surface $X_{\left[I V^{*}, 3,1\right]}$, at which two fibers of type $I_{3}$ and two fibers of type $I_{1}$ collide, gives K3 surface with singular fibers of types $I V^{*}, I V^{*}, I_{6}$, and $I_{2}$.

For the gluing of two extremal rational elliptic surfaces $X_{\left[4^{*}, 1,1\right]}$, when we consider the limit of the quadratic base change at which two pairs of type $I_{1}$ fibers collide, the resulting K3 surface has two singular fibers of type $I_{4}^{*}$ and two singular fibers of type $I_{2}$. For extremal rational elliptic surface $X_{\left[2^{*}, 2,2\right]}$, when we consider the limit of the quadratic base change at which two pairs of type $I_{2}$ fibers collide, the resulting K3 surface has two type $I_{2}^{*}$ fibers and two type $I_{4}$ fibers. For the gluing of two extremal rational elliptic surface $X_{\left[1^{*}, 4,1\right]}$, when we consider the limit of the quadratic base change at which two type $I_{4}$ fibers and two type $I_{1}$ fibers collide, the resulting $\mathrm{K} 3$ surface has type $I_{1}^{*}, I_{1}^{*}, I_{8}$, and $I_{2}$ fibers. 
For extremal rational elliptic surface $X_{[5,5,1,1]}$, when we consider the limit of the quadratic base change at which two fibers of type $I_{5}$ and two fibers of type $I_{1}$ collide, the resulting K3 surface has 1 type $I_{10}$ fiber, 2 type $I_{5}$ fibers, 1 type $I_{2}$ fiber, and 2 type $I_{1}$ fibers.

From $A D E$ types of the $10 \mathrm{~K} 3$ surfaces that we obtained above as the quadratic base change of extremal rational elliptic surfaces, we conclude that they are extremal K3 surfaces. $A D E$ types of the extremal K3 surfaces and the corresponding complex structures were classified in [25]. Mordell-Weil groups of extremal K3 surfaces were also derived in [25]. Using table 2 in [25], we can deduce the complex structures of the 10 extremal K3 surfaces from their $A D E$ types.

The complex structure of an attractive K3 surface $S$ is specified by the transcendental lattice $T(S)$, which is defined to be the orthogonal complement of the Néron-Severi lattice in the K3 lattice $H^{2}(S, \mathbb{Z})[26]$. Therefore, we represent the complex structure of an attractive K3 surface by the intersection matrix of the transcendental lattice in this study. For an attractive K3 surface, the transcendental lattice is a $2 \times 2$ integral, symmetric, positivedefinite even lattice. See section 4 in [24] for a review of the correspondence of the complex structures of attractive K3 surfaces and the transcendental lattice.

We list the fiber types, corresponding $A D E$ types, the complex structures and the Mordell-Weil groups of the 10 extremal K3 surfaces, that we obtained above as deformation of two isomorphic extremal rational elliptic surfaces glued together, in table 4. The global structures of the non-Abelian gauge symmetries ${ }^{3}$ that arise on the 7-branes in Ftheory compactifications on the 10 extremal K3 surfaces times a K3 surface are also shown in table 4. Extremal K3 surface has the Mordell-Weil rank 0, therefore these F-theory compactifications do not have $\mathrm{U}(1)$ gauge field.

\subsubsection{Weierstrass equation}

We obtained 10 extremal K3 surfaces in section 2.4.1 as the limit of the quadratic base change $^{4}$ of extremal rational elliptic surfaces, at which singular fibers of the same type collide. Therefore, by substituting appropriate quadratic polynomials into $u, v$ in the Weierstrass forms of extremal rational elliptic surfaces, listed in table 1 in section 2.2 , we can deduce the Weierstrass forms of the 10 extremal K3 surfaces, obtained in section 2.4.1.

As an example, extremal K3 surface whose transcendental lattice has the intersection matrix $\left(\begin{array}{ll}6 & 0 \\ 0 & 2\end{array}\right)$, with $A D E$ type $E_{6}^{2} \oplus A_{5} \oplus A_{1}$, is obtained via the quadratic base change of extremal rational elliptic surface $X_{\left[I V^{*}, 3,1\right]}$, in which two fibers of type $I_{3}$ and two fibers of type $I_{1}$ collide. The Weierstrass form of extremal rational elliptic surface $X_{\left[I V^{*}, 3,1\right]}$ is given by

$$
y^{2}=x^{3}+v^{3}(24 u-27 v) x+v^{4}\left(16 u^{2}-72 u v+54 v^{2}\right) .
$$

The discriminant of the Weierstrass form $(2.15)$ is [18]

$$
\Delta \sim u^{3} v^{8}(u-v)
$$

\footnotetext{
${ }^{3}$ See $[8,27]$ for the correspondence of the types of the singular fibers and the non-Abelian gauge groups on the 7-branes.

${ }^{4}$ The relationship of K3 surfaces and rational elliptic surfaces via base change is also discussed in [28].
} 


\begin{tabular}{|c|c|c|c|c|}
\hline Singular fibers & $A D E$ type & Complex Str. & Mordell-Weil group & Gauge group \\
\hline$I I^{*} I I^{*} I V$ & $E_{8}^{2} \oplus A_{2}$ & $\left(\begin{array}{ll}2 & 1 \\
1 & 2\end{array}\right)$ & 0 & $E_{8}^{2} \times \mathrm{SU}(3)$ \\
\hline$I I I^{*} I I I^{*} I_{0}^{*}$ & $E_{7}^{2} \oplus D_{4}$ & $\left(\begin{array}{ll}2 & 0 \\
0 & 2\end{array}\right)$ & $\mathbb{Z}_{2}$ & $E_{7}^{2} \times \mathrm{SO}(8) / \mathbb{Z}_{2}$ \\
\hline$I V^{*} I V^{*} I V^{*}$ & $E_{6}^{3}$ & $\left(\begin{array}{ll}2 & 1 \\
1 & 2\end{array}\right)$ & $\mathbb{Z}_{3}$ & $E_{6}^{3} / \mathbb{Z}_{3}$ \\
\hline$I I^{*} I I^{*} I_{2} I_{2}$ & $E_{8}^{2} \oplus A_{1}^{2}$ & $\left(\begin{array}{ll}2 & 0 \\
0 & 2\end{array}\right)$ & 0 & $E_{8}^{2} \times \mathrm{SU}(2)^{2}$ \\
\hline$I I I^{*} I I I^{*} I_{4} I_{2}$ & $E_{7}^{2} \oplus A_{3} \oplus A_{1}$ & $\left(\begin{array}{ll}4 & 0 \\
0 & 2\end{array}\right)$ & $\mathbb{Z}_{2}$ & $E_{7}^{2} \times \mathrm{SU}(4) \times \mathrm{SU}(2) / \mathbb{Z}_{2}$ \\
\hline$I V^{*} I V^{*} I_{6} I_{2}$ & $E_{6}^{2} \oplus A_{5} \oplus A_{1}$ & $\left(\begin{array}{ll}6 & 0 \\
0 & 2\end{array}\right)$ & $\mathbb{Z}_{3}$ & $E_{6}^{2} \times \mathrm{SU}(6) \times \mathrm{SU}(2) / \mathbb{Z}_{3}$ \\
\hline$I_{4}^{*} I_{4}^{*} I_{2} I_{2}$ & $D_{8}^{2} \oplus A_{1}^{2}$ & $\left(\begin{array}{ll}2 & 0 \\
0 & 2\end{array}\right)$ & $\mathbb{Z}_{2} \times \mathbb{Z}_{2}$ & $\mathrm{SO}(16)^{2} \times \mathrm{SU}(2)^{2} / \mathbb{Z}_{2} \times \mathbb{Z}_{2}$ \\
\hline$I_{2}^{*} I_{2}^{*} I_{4} I_{4}$ & $D_{6}^{2} \oplus A_{3}^{2}$ & $\left(\begin{array}{ll}4 & 0 \\
0 & 4\end{array}\right)$ & $\mathbb{Z}_{2} \times \mathbb{Z}_{2}$ & $\mathrm{SO}(12)^{2} \times \mathrm{SU}(4)^{2} / \mathbb{Z}_{2} \times \mathbb{Z}_{2}$ \\
\hline$I_{1}^{*} I_{1}^{*} I_{8} I_{2}$ & $D_{5}^{2} \oplus A_{7} \oplus A_{1}$ & $\left(\begin{array}{ll}8 & 0 \\
0 & 2\end{array}\right)$ & $\mathbb{Z}_{4}$ & $\mathrm{SO}(10)^{2} \times \mathrm{SU}(8) \times \mathrm{SU}(2) / \mathbb{Z}_{4}$ \\
\hline$I_{10} \quad I_{5} I_{5} I_{2} I_{1} I_{1}$ & $A_{9} \oplus A_{4}^{2} \oplus A_{1}$ & $\left(\begin{array}{cc}10 & 0 \\
0 & 2\end{array}\right)$ & $\mathbb{Z}_{5}$ & $\mathrm{SU}(10) \times \mathrm{SU}(5)^{2} \times \mathrm{SU}(2) / \mathbb{Z}_{5}$ \\
\hline
\end{tabular}

Table 4. Configurations of singular fibers, $A D E$ types, complex structures and the Mordell-Weil groups of 10 extremal K3 surfaces. We also list the global structures of the non-Abelian gauge groups that form on the 7 -branes.

Type $I V^{*}$ fiber is at $[u: v]=[1: 0]$, type $I_{3}$ fiber is at $[u: v]=[0: 1]$ and type $I_{1}$ fiber is at $[u: v]=[1: 1]$. We consider the following substitutions for $u, v$ in the Weierstrass form of extremal rational elliptic surface $X_{\left[I V^{*}, 3,1\right]}$ :

$$
\begin{aligned}
& u=\tilde{u}^{2} \\
& v=2 \tilde{u} \tilde{v}-\tilde{v}^{2} .
\end{aligned}
$$

This gives the limit of the quadratic base change at which two fibers of type $I_{3}$ collide at $[u: v]=[0: 1]$ and two fibers of type $I_{1}$ collide at $[u: v]=[1: 1]$. The resulting equation

$$
\begin{aligned}
y^{2}= & x^{3}+v^{3}(2 u-v)^{3}\left(24 u^{2}+27 v^{2}-54 u v\right) x \\
& +v^{4}(2 u-v)^{4}\left(16 u^{4}-144 u^{3} v+288 u^{2} v^{2}-216 u v^{3}+54 v^{4}\right)
\end{aligned}
$$

gives the Weierstrass form of extremal K3 surface with transcendental lattice $\left(\begin{array}{ll}6 & 0 \\ 0 & 2\end{array}\right)$, with $A D E$ type $E_{6}^{2} \oplus A_{5} \oplus A_{1}$. The discriminant of the Weierstrass form (2.18) is given by

$$
\Delta \sim u^{6} v^{8}(2 u-v)^{8}(u-v)^{2} .
$$

We confirm from equations (2.18) and (2.19) that extremal K3 surface (2.18) has two type $I V^{*}$ fibers, at $[u: v]=[1: 0],[1: 2]$, type $I_{6}$ fiber at $[u: v]=[0: 1]$ and type $I_{2}$ fiber at $[u: v]=[1: 1]$. 


\begin{tabular}{|c|c|c|}
\hline$A D E$ type & $f$ & $g$ \\
\hline$E_{8}^{2} \oplus A_{2}$ & 0 & $(u-v)^{2} u^{5} v^{5}$ \\
\hline$E_{7}^{2} \oplus D_{4}$ & $(u-v)^{2} u^{3} v^{3}$ & 0 \\
\hline$E_{6}^{3}$ & 0 & $(u-v)^{4} u^{4} v^{4}$ \\
\hline$E_{8}^{2} \oplus A_{1}^{2}$ & $-3 u^{4} v^{4}$ & $u^{5} v^{5}\left(u^{2}+v^{2}\right)$ \\
\hline$E_{7}^{2} \oplus A_{3} \oplus A_{1}$ & $-\frac{9}{16}\left(u^{2}+v^{2}+\frac{10}{3} u v\right) u^{3} v^{3}$ & $\frac{9}{4} u^{5} v^{5}\left(\frac{1}{4} u^{2}+\frac{1}{4} v^{2}+\frac{7}{18} u v\right)$ \\
\hline$E_{6}^{2} \oplus A_{5} \oplus A_{1}$ & $v^{3}(2 u-v)^{3}\left(24 u^{2}+27 v^{2}-54 u v\right)$ & $v^{4}(2 u-v)^{4} \cdot\left(16 u^{4}-144 u^{3} v\right.$ \\
\hline$D_{8}^{2} \oplus A_{1}^{2}$ & $-3 u^{2} v^{2}\left(u^{4}+v^{4}-u^{2} v^{2}\right)$ & $\left.+288 u^{2} v^{2}-216 u v^{3}+54 v^{4}\right)$ \\
\hline$D_{6}^{2} \oplus A_{3}^{2}$ & $-3 \cdot \frac{4}{\omega(\omega-1)} u v\left[u^{2}+v^{2}+\left(2+\frac{4}{\omega-1}\right) u v\right]$ & {$\left[u^{2}+v^{2}\right) u^{3} v^{3}\left(2 u^{4}-5 u^{2} v^{2}+2 v^{4}\right)$} \\
\hline$D_{5}^{2} \oplus A_{7} \oplus A_{1}$ & $-\left[u^{2}+v^{2}+\left(2+\frac{4}{\omega}\right) u v\right]^{2}$ & $\cdot\left\{\left[u^{2}+v^{2}+\left(2+\frac{4}{\omega}\right) u v\right]^{3}\right.$ \\
& $-3\left(u^{4}+4 u^{3} v+4 u^{2} v^{2}-\frac{3}{4} v^{4}\right)$ & $u(u+2 v)\left(2 u^{4}+8 u^{3} v+8 u^{2} v^{2}-\frac{9}{4} v^{4}\right)$ \\
& $\cdot\left(u^{2}+2 u v-v^{2}\right)^{2}$ & $\cdot\left(u^{2}+2 u v-v^{2}\right)^{3}$ \\
\hline & $-3\left[u^{8}-12 u^{6} \cdot \frac{2}{11+5 \sqrt{5}}\left(2 u v-v^{2}\right)\right.$ & $2\left[u^{32}-18 u^{10} \cdot \frac{2}{11+5 \sqrt{5}}\left(2 u v-v^{2}\right)\right.$ \\
& $+14 u^{4}\left(\frac{2}{11+5 \sqrt{5}}\left(2 u v-v^{2}\right)\right)^{2}$ & $+75 u^{8}\left(\frac{2}{11+5 \sqrt{5}}\left(2 u v-v^{2}\right)\right)^{2}$ \\
$A_{9} \oplus A_{4}^{2} \oplus A_{1}$ & $+12 u^{2}\left(\frac{2}{11+5 \sqrt{5}}\left(2 u v-v^{2}\right)\right)^{3}$ & $+75 u^{4}\left(\frac{2}{11+5 \sqrt{5}}\left(2 u v-v^{2}\right)\right)^{4}$ \\
& $\left.+\left(\frac{2}{11+5 \sqrt{5}}\left(2 u v-v^{2}\right)\right)^{4}\right]$ & $+18 u^{2}\left(\frac{2}{11+5 \sqrt{5}}\left(2 u v-v^{2}\right)\right)^{5}$ \\
& \multicolumn{2}{c}{$\left.+\left(\frac{2}{11+5 \sqrt{5}}\left(2 u v-v^{2}\right)\right)^{6}\right]$} \\
\hline
\end{tabular}

Table 5. $A D E$ types of extremal K3 surfaces, and coefficients $f, g$ of the Weierstrass form $y^{2}=$ $x^{3}+f x+g .[u: v]$ denotes the homogeneous coordinate on the base $\mathbb{P}^{1} . \omega$ denotes a cube root of unity, $\omega \neq 1$.

We show the Weierstrass forms of the 10 extremal K3 surfaces, which we obtained in section 2.4.1, in table 5. The discriminants of the Weierstrass forms in table 5 are listed in table 6. The Weierstrass forms of extremal K3 surfaces with $A D E$ types $E_{8}^{2} \oplus A_{2}$ and $E_{8}^{2} \oplus A_{1}^{2}$ are discussed in [22]. The Weierstrass forms of extremal K3 surfaces with $A D E$ types $E_{6}^{3}$ and $E_{7}^{2} \oplus D_{4}$ are discussed in [24] and [23], respectively.

\subsubsection{Anomaly cancellation condition}

We consider F-theory compactifications on the extremal K3 surfaces obtained in section 2.4.1 times a K3 surface. The resulting theory is a four-dimensional theory with $N=2$, without a four-form flux. The anomaly cancellation condition determines the form of the discriminant locus to be $24 \mathrm{~K} 3$ surfaces, counted with multiplicity; there are 24 7-branes wrapped on the K3 surfaces. ${ }^{5}$ We show the correspondence of the numbers of 7 -branes and the fiber types in table 7 . The Euler numbers of the singular fibers can be found in [29]. The Euler number of fiber type can be considered as the number of the associated 7-branes. We confirm from tables 4 and 7 that there are in fact 24 7-branes in

\footnotetext{
${ }^{5}$ See, for example, [24] for discussion.
} 


\begin{tabular}{|c|c|}
\hline$A D E$ type & $\Delta$ \\
\hline$E_{8}^{2} \oplus A_{2}$ & $(u-v)^{4} u^{10} v^{10}$ \\
\hline$E_{7}^{2} \oplus D_{4}$ & $(u-v)^{6} u^{9} v^{9}$ \\
\hline$E_{6}^{3}$ & $(u-v)^{8} u^{8} v^{8}$ \\
\hline$E_{8}^{2} \oplus A_{1}^{2}$ & $u^{10} v^{10}(u-v)^{2}(u+v)^{2}$ \\
\hline$E_{7}^{2} \oplus A_{3} \oplus A_{1}$ & $u^{9} v^{9}(u-v)^{4}(u+v)^{2}$ \\
\hline$E_{6}^{2} \oplus A_{5} \oplus A_{1}$ & $u^{6} v^{8}(2 u-v)^{8}(u-v)^{2}$ \\
\hline$D_{8}^{2} \oplus A_{1}^{2}$ & $u^{10} v^{10}(u-v)^{2}(u+v)^{2}$ \\
\hline$D_{6}^{2} \oplus A_{3}^{2}$ & $(u-v)^{4}(u+v)^{4}\left[u^{2}+v^{2}+\left(2+\frac{4}{\omega}\right) u v\right]^{8}$ \\
\hline$D_{5}^{2} \oplus A_{7} \oplus A_{1}$ & $v^{8}\left(u^{2}+2 u v-v^{2}\right)^{7}(u+v)^{2}$ \\
\hline$A_{9} \oplus A_{4}^{2} \oplus A_{1}$ & $u^{10} v^{5}(v-2 u)^{5}(u-v)^{2}$ \\
\hline
\end{tabular}

Table 6. Discriminant $\Delta$ of the Weierstrass forms in table 5 are shown. We suppressed the irrelevant constant factors of the discriminants.

\begin{tabular}{|c|c|}
\hline Fiber type & \# of 7-branes (Euler number) \\
\hline$I_{n}$ & $n$ \\
$I_{0}^{*}$ & 6 \\
$I_{m}^{*}$ & $m+6$ \\
$I I$ & 2 \\
$I I I$ & 3 \\
$I V$ & 4 \\
$I V^{*}$ & 8 \\
$I I I^{*}$ & 9 \\
$I I^{*}$ & 10 \\
\hline
\end{tabular}

Table 7. Associated numbers of 7-branes for fiber types.

F-theory compactifications on the 10 extremal K3 surfaces times a K3 surface. Therefore, we conclude that the anomaly cancellation condition is satisfied for these compactifications.

By turning on four-form flux [30-34], F-theory compactification on K3 times K3 gives four-dimensional theory with $N=1$ supersymmetry. We confirm from table 1 in [35] and table 2 in [13] that for F-theory compactifications on the 10 extremal K3 surfaces, obtained in section 2.4.1, times some appropriate attractive K3 surface, the tadpole [31] can be cancelled. See $[23,24]$ for the details. 


\section{Stable degeneration of K3 to a pair of non-isomorphic rational elliptic surfaces}

In this section, we discuss the stable degeneration where a K3 surface degenerates into two non-isomorphic rational elliptic surfaces. It is considerably difficult to provide a general equation to describe this kind of stable degeneration. Therefore, instead of providing an equation to describe the process, we use a lattice theoretic approach to determine whether stable degeneration exists for pairs of non-isomorphic rational elliptic surfaces. For the sake of brevity, in this section, we simply say "a pair of rational elliptic surfaces" to indicate a pair of non-isomorphic rational elliptic surfaces. We also discuss the configurations of singular fibers under stable degeneration. In section 3.1, we will discuss a lattice theoretic condition for the existence of stable degeneration for pairs of rational elliptic surfaces. Applying the lattice condition, in section 3.2, we demonstrate that stable degeneration exists for pairs of extremal rational elliptic surfaces.

Elliptic fiber of a rational elliptic surface generally has the moduli of dimension 1 over the base $\mathbb{P}^{1}$. Therefore, given a pair of rational elliptic surfaces, there is a pair of isomorphic smooth elliptic fibers, and the pair of rational elliptic surfaces can be glued along the isomorphic smooth fibers. However, when elliptic fiber of a rational elliptic surface has the constant moduli over the base $\mathbb{P}^{1}$, such gluing is not necessarily possible. For the three extremal rational elliptic surfaces $X_{\left[I I, I I^{*}\right]}, X_{\left[I I I, I I I^{*}\right]}, X_{\left[I V, I V^{*}\right]}$, the complex structure of elliptic fibers is constant over the base $\mathbb{P}^{1}{ }^{6}$ We do not consider the three extremal rational elliptic surfaces $X_{\left[I I, I I^{*}\right]}, X_{\left[I I I, I I I^{*}\right]}, X_{\left[I V, I V^{*}\right]}$ in this section.

\subsection{Lattice condition for stable degeneration limit}

We use the Torelli theorem for K3 surfaces [36] to deduce a lattice theoretic condition that determines whether pairs of rational elliptic surfaces admit stable degeneration. The Torelli theorem for K3 surfaces states that the geometry of a K3 surface is determined by the structure of the K3 lattice $\Lambda_{\mathrm{K} 3}$. The K3 lattice $\Lambda_{\mathrm{K} 3}$ of a K3 surface $S$ is the second integral cohomology group $H^{2}(S, \mathbb{Z})$. The $\mathrm{K} 3$ lattice $\Lambda_{\mathrm{K} 3}$ is the indefinite even unimodular lattice of signature $(3,19)$, and it is the direct sum of three copies of the hyperbolic plane $U$ and two copies of the $E_{8}$ lattice:

$$
\Lambda_{\mathrm{K} 3} \cong U^{3} \oplus E_{8}^{2}
$$

In this note, we assume that a rational elliptic surface has a global section; thus, we presume that the K3 surface obtained as a deformation of the sum of two rational elliptic surfaces also admits a global section. ${ }^{7}$ An elliptic K3 surface having a section is equivalent

\footnotetext{
${ }^{6}$ The complex structure of elliptic fibers of extremal rational elliptic surface $X_{\left[0^{*}, 0^{*}\right]}(j)$ is also constant over the base $\mathbb{P}^{1}$, but there is a degree of freedom in choosing j-invariant of an elliptic fiber. Therefore, when j-invariant is appropriately chosen, $X_{\left[0^{*}, 0^{*}\right]}(j)$ can be glued with another rational elliptic surface. For this reason, we include extremal rational elliptic surface $X_{\left[0^{*}, 0^{*}\right]}(j)$.

${ }^{7}$ In general, genus-one fibered K3 surfaces need not have a global section. For discussion of the geometry of genus-one fibered K3 surfaces without a section and string compactifications on such spaces, see, for example, [23, 24, 37]. For recent progress in F-theory compactifications on genus-one fibrations without a section, see, for example, also [38-48].
} 
to the primitive embedding of the hyperbolic plane $U$ into the K3 lattice $\Lambda_{\mathrm{K} 3}[36,49]$. We denote the $A D E$ types of two rational elliptic surfaces, $X_{1}$ and $X_{2}$, as $R_{1}$ and $R_{2}$, respectively. Applying the argument in [50] to the boundary of the closure of K3 moduli, from the Torelli theorem for K3 surfaces, we deduce that a K3 surface exists and it admits stable degeneration into two rational elliptic surfaces $X_{1}$ and $X_{2}$, exactly when there is a primitive embedding of the lattice $U \oplus R_{1} \oplus R_{2}$ into the K3 lattice $\Lambda_{\mathrm{K} 3}$ :

$$
U \oplus R_{1} \oplus R_{2} \subset \Lambda_{\mathrm{K} 3} .
$$

Whether lattice $U \oplus R_{1} \oplus R_{2}$ primitively embeds into the K3 lattice $\Lambda_{\mathrm{K} 3}$ can be determined by the criterion given in [51]. Some lattice theoretic terms are necessary to state the criterion; we introduce some lattice theoretic terms first.

By lattice, we indicate a finite rank free $\mathbb{Z}$-module with a non-degenerate integral symmetric bilinear form. The lattice $L$ is said to be even, when for every element $x$ of $L$, $x^{2}=x \cdot x$ is even. The discriminant of lattice $L$, disc $L$, is the determinant of an intersection matrix $\left(e_{i} \cdot e_{j}\right)_{i j}$ for a basis $\left\{e_{i}\right\}$ of the lattice $L$. The lattice $L$ is said to be unimodular when its discriminant is \pm 1 . $U$ denotes the hyperbolic plane. The hyperbolic plane $U$ is the even unimodular lattice of signature $(1,1) . E_{8}$ denotes the even unimodular lattice of signature $(0,8) . E_{8}$ and $U$ are unique up to the isometries of lattice. When the lattice $L_{1}$ embeds into the lattice $L_{2}$, the embedding $L_{1} \subset L_{2}$ is said to be primitive when the quotient $L_{2} / L_{1}$ is free as a $\mathbb{Z}$-module. The dual lattice of lattice $L$ is the lattice $\operatorname{Hom}(L, \mathbb{Z})$, and is denoted as $L^{*}$. The quotient $G_{L}:=L^{*} / L$ is a finite Abelian group, and this group is called the discriminant group. When $L$ is an even lattice, the map $q_{L}: G_{L} \rightarrow \mathbb{Q} / 2 \mathbb{Z}$,

$$
q_{L}(x)=x^{2} \bmod 2 \mathbb{Z}
$$

defines a non-degenerate quadratic form of the discriminant group $G_{L}$; form $q_{L}$ is called the discriminant form.

When $A$ is a finite Abelian group, its length $l(A)$ is defined to be the minimum number of elements required to generate group $A$.

Further, we state the criterion of primitive lattice embedding.

Criterion $(C)$ [51]. $\quad M$ is an even lattice of signature $\left(m_{+}, m_{-}\right), G$ is the discriminant group of $M$ and $q$ is the discriminant form of $M$. Then, $M$ primitively embeds into some even unimodular lattice of signature $\left(l_{+}, l_{-}\right)$when all of the three conditions i)-iii) are satisfied:

i) $l_{+}+l_{-}-\operatorname{rk} M>l(G)$.

ii) $l_{+}-m_{+} \geq 0, \quad l_{-}-m_{-} \geq 0$.

iii) $l_{+}-l_{-} \equiv 0(\bmod 8)$

\subsection{Pairs of extremal rational elliptic surfaces}

Applying criterion $(C)$, we discuss whether the lattice $U \oplus R_{1} \oplus R_{2}$ of pairs of extremal rational elliptic surfaces, $X_{1} \amalg X_{2}$, primitively embeds into the K3 lattice $\Lambda_{\mathrm{K} 3}$. This 


\begin{tabular}{|c|c|c|}
\hline Extremal rational elliptic surface & $A D E$ type & Discriminant group \\
\hline$X_{\left[0^{*}, 0^{*}\right]}(j)$ & $D_{4}^{2}$ & $\mathbb{Z} / 2 \mathbb{Z} \times \mathbb{Z} / 2 \mathbb{Z}$ \\
\hline$X_{\left[I I^{*}, 1,1\right]}$ & $E_{8}$ & 0 \\
\hline$X_{\left[I I I^{*}, 2,1\right]}$ & $E_{7} \oplus A_{1}$ & $\mathbb{Z} / 2 \mathbb{Z}$ \\
\hline$X_{\left[I V^{*}, 3,1\right]}$ & $E_{6} \oplus A_{2}$ & $\mathbb{Z} / 3 \mathbb{Z}$ \\
\hline$X_{\left[4^{*}, 1,1\right]}$ & $D_{8}$ & $\mathbb{Z} / 2 \mathbb{Z}$ \\
\hline$X_{\left[2^{*}, 2,2\right]}$ & $D_{6} \oplus A_{1}^{2}$ & $\mathbb{Z} / 2 \mathbb{Z} \times \mathbb{Z} / 2 \mathbb{Z}$ \\
\hline$X_{\left[1^{*}, 4,1\right]}$ & $D_{5} \oplus A_{3}$ & $\mathbb{Z} / 4 \mathbb{Z}$ \\
\hline$X_{[9,1,1,1]}$ & $A_{8}$ & $\mathbb{Z} / 3 \mathbb{Z}$ \\
\hline$X_{[8,2,1,1]}$ & $A_{7} \oplus A_{1}$ & $\mathbb{Z} / 4 \mathbb{Z}$ \\
\hline$X_{[6,3,2,1]}$ & $A_{5} \oplus A_{2} \oplus A_{1}$ & $\mathbb{Z} / 6 \mathbb{Z}$ \\
\hline$X_{[5,5,1,1]}$ & $A_{4}^{2}$ & $\mathbb{Z} / 5 \mathbb{Z}$ \\
\hline$X_{[4,4,2,2]}$ & $A_{3}^{2} \oplus A_{1}^{2}$ & $\mathbb{Z} / 4 \mathbb{Z} \times \mathbb{Z} / 2 \mathbb{Z}$ \\
\hline$X_{[3,3,3,3]}$ & $A_{2}^{4}$ & $\mathbb{Z} / 3 \mathbb{Z} \times \mathbb{Z} / 3 \mathbb{Z}$ \\
\hline & & \\
\hline
\end{tabular}

Table 8. The discriminant groups and $A D E$ types of the extremal rational elliptic surfaces.

determines whether stable degeneration exists for pairs of extremal rational elliptic surfaces. An even unimodular lattice of signature $(3,19)$ is unique up to the isometries of lattice [52]. Therefore, when the lattice $U \oplus R_{1} \oplus R_{2}$ admits a primitive embedding into some even unimodular lattice of signature $(3,19)$, it primitively embeds into the K3 lattice $\Lambda_{\mathrm{K} 3}$.

As stated in section 2.2, the complex structures and singular fiber types of the extremal rational elliptic surfaces were classified. For an extremal rational elliptic surface, the discriminant group and the Mordell-Weil group are identical [53]. The Mordell-Weil groups of the rational elliptic surfaces were computed in [54]. We list the discriminant groups and $A D E$ types of extremal rational elliptic surfaces in table 8.

Let $G$ denote the product group of the discriminant groups of two extremal rational elliptic surfaces $X_{1}$ and $X_{2}$. We determine the pairs of extremal rational elliptic surfaces that satisfy the condition i)

$$
\operatorname{rk} \Lambda_{\mathrm{K} 3}-\operatorname{rk} U \oplus R_{1} \oplus R_{2}>l(G) .
$$

in criterion $(C)$. From table 8, we observe that the length of the discriminant group of an extremal rational elliptic surface is either 1 or 2 . For two extremal rational elliptic surfaces, $X_{1}$ and $X_{2}$, the singular fiber types $R_{1}$ and $R_{2}$ are rank 8 even lattices

$$
\operatorname{rk} R_{1}=\operatorname{rk} R_{2}=8
$$


of signature $(0,8)$. Thus, the lattice $U \oplus R_{1} \oplus R_{2}$ has the signature $(1,17)$. The difference of the rank of the K3 lattice $\Lambda_{\mathrm{K} 3}$ and the rank of the lattice $U \oplus R_{1} \oplus R_{2}$ is

$$
3+19-(1+17)=4 .
$$

The length $l(G)$ of the discriminant group $G$ attains this bound only when the discriminant groups of two extremal rational elliptic surfaces both have the length 2. The product group $G$ of the discriminant groups of extremal rational elliptic surfaces has the length 4 only for three pairs ${ }^{8}$ of extremal rational elliptic surfaces:

$$
X_{[4,4,2,2]} \amalg X_{\left[2^{*}, 2,2\right]}, \quad X_{\left[2^{*}, 2,2\right]} \amalg X_{\left[0^{*}, 0^{*}\right]}, \quad X_{\left[0^{*}, 0^{*}\right]} \amalg X_{[4,4,2,2]} .
$$

For all other pairs of extremal rational elliptic surfaces, the criterion $(C)$ applies. Both conditions ii) and iii) in criterion $(C)$ are satisfied:

$$
3-1>0,19-17>0,
$$

and

$$
3-19 \equiv 0(\bmod 8)
$$

Thus, we determine that the lattice $U \oplus R_{1} \oplus R_{2}$ primitively embeds into the K3 lattice $\Lambda_{\mathrm{K} 3}$ for all pairs of extremal rational elliptic surfaces, except the three pairs $X_{[4,4,2,2]} \amalg X_{\left[2^{*}, 2,2\right]}$, $X_{\left[2^{*}, 2,2\right]} \amalg X_{\left[0^{*}, 0^{*}\right]}$ and $X_{\left[0^{*}, 0^{*}\right]} \amalg X_{[4,4,2,2]}$. We conclude that the stable degeneration of a K3 surface exists for all pairs of extremal rational elliptic surfaces except the three pairs.

The remaining three pairs, $X_{[4,4,2,2]} \amalg X_{\left[2^{*}, 2,2\right]}, X_{\left[2^{*}, 2,2\right]} \amalg X_{\left[0^{*}, 0^{*}\right]}$ and $X_{\left[0^{*}, 0^{*}\right]} \amalg$ $X_{[4,4,2,2]}$, have the $A D E$ types $D_{6} \oplus A_{3}^{2} \oplus A_{1}^{4}, D_{6} \oplus D_{4}^{2} \oplus A_{1}^{2}, D_{4}^{2} \oplus A_{3}^{2} \oplus A_{1}^{2}$, respectively. $A D E$ types of the singular fibers of elliptic K3 surfaces with a global section were classified in [55]. We conclude from table 1 in [55] that the lattice $U \oplus R_{1} \oplus R_{2}$ primitively embeds into the K3 lattice $\Lambda_{\mathrm{K} 3}$ for the three pairs of extremal rational elliptic surfaces. (They correspond to No.2079, 2043, 2152 in table 1 in [55], respectively.) This demonstrates that the stable degeneration of a K3 surface exists for the remaining three pairs of extremal rational elliptic surfaces.

The aforementioned argument demonstrates that stable degeneration exists for all pairs $^{9}$ of extremal rational elliptic surfaces.

From lattice embedding

$$
U \oplus R_{1} \oplus R_{2} \subset \Lambda_{\mathrm{K} 3},
$$

we deduce that the $A D E$ type of the singular fibers of the resulting K3 surface is the sum of the $A D E$ types of the singular fibers of the two non-isomorphic extremal rational elliptic surfaces.

\footnotetext{
${ }^{8}$ The product of discriminant group $\mathbb{Z} / 3 \mathbb{Z} \times \mathbb{Z} / 3 \mathbb{Z}$ and another discriminant group with length 2 has length 2 . For example, the product of $\mathbb{Z} / 3 \mathbb{Z} \times \mathbb{Z} / 3 \mathbb{Z}$ with $\mathbb{Z} / 2 \mathbb{Z} \times \mathbb{Z} / 2 \mathbb{Z}$ is isomorphic to $\mathbb{Z} / 6 \mathbb{Z} \times \mathbb{Z} / 6 \mathbb{Z}$, which has the length 2 .

${ }^{9}$ As we stated at the beginning of this section, the three extremal rational elliptic surfaces $X_{\left[I I, I I^{*}\right]}$, $X_{\left[I I I, I I I^{*}\right]}, X_{\left[I V, I V^{*}\right]}$ are not considered in this section.
} 


\section{Conclusion}

In this study, we analyzed the stable degeneration of a K3 surface into two rational elliptic surfaces. We also discussed the configurations of singular fibers under the stable degeneration limit.

We demonstrated that gluing together two isomorphic rational elliptic surfaces and deforming the resulting surface to a K3 surface is always possible. We provided an equation to describe this kind of stable degeneration. The sum of two isomorphic rational elliptic surfaces glued together admits a deformation to a K3 surface, the singular fibers of which are twice the singular fibers of the rational elliptic surface. For special cases, two fibers of the same type of the resulting K3 surface collide, and they are enhanced to a fiber of another type. Some K3 surfaces become attractive in these cases. We determined the complex structures and the Weierstrass forms of these attractive K3 surfaces. We also deduced the gauge groups in F-theory compactifications on these attractive K3 surfaces times a K3.

We also investigated the deformation of two non-isomorphic rational elliptic surfaces glued together to a K3 surface, using the Torelli theorem of K3 surfaces. We deduced the lattice theoretic condition that must be satisfied to ensure that a deformation to a K3 surface exists for pairs of non-isomorphic rational elliptic surfaces. We confirmed that the lattice condition is satisfied for all pairs of the extremal rational elliptic surfaces. Thus, all such pairs of extremal rational elliptic surfaces glued together admit a deformation to a K3 surface. This demonstrates that for any pair of extremal rational elliptic surfaces, except the three extremal rational elliptic surfaces $X_{\left[I I, I I^{*}\right]}, X_{\left[I I I, I I I^{*}\right]}, X_{\left[I V, I V^{*}\right]}$, there is a K3 surface that stably degenerates into that pair. The $A D E$ type of singular fibers of the resulting $\mathrm{K} 3$ surface is the sum of those of the two non-isomorphic extremal rational elliptic surfaces glued together. The lattice condition discussed in this study can be extended to general pairs of rational elliptic surfaces.

\section{Acknowledgments}

We would like to thank Shun'ya Mizoguchi and Shigeru Mukai for discussions. This work is partially supported by Grant-in-Aid for Scientific Research \#16K05337 from The Ministry of Education, Culture, Sports, Science and Technology of Japan.

Open Access. This article is distributed under the terms of the Creative Commons Attribution License (CC-BY 4.0), which permits any use, distribution and reproduction in any medium, provided the original author(s) and source are credited.

\section{References}

[1] E. Witten, Strong coupling expansion of Calabi-Yau compactification, Nucl. Phys. B 471 (1996) 135 [hep-th/9602070] [INSPIRE].

[2] R. Donagi and M. Wijnholt, Model building with F-theory, Adv. Theor. Math. Phys. 15 (2011) 1237 [arXiv: 0802 .2969] [INSPIRE]. 
[3] C. Beasley, J.J. Heckman and C. Vafa, GUTs and exceptional branes in F-theory - I, JHEP 01 (2009) 058 [arXiv:0802.3391] [inSPIRE].

[4] C. Beasley, J.J. Heckman and C. Vafa, GUTs and exceptional branes in F-theory - II: experimental predictions, JHEP 01 (2009) 059 [arXiv:0806.0102] [INSPIRE].

[5] R. Donagi and M. Wijnholt, Breaking GUT groups in F-theory, Adv. Theor. Math. Phys. 15 (2011) 1523 [arXiv: 0808.2223] [inSPIRE].

[6] C. Vafa, Evidence for F-theory, Nucl. Phys. B 469 (1996) 403 [hep-th/9602022] [InSPIRE].

[7] D.R. Morrison and C. Vafa, Compactifications of F-theory on Calabi-Yau threefolds. 1, Nucl. Phys. B 473 (1996) 74 [hep-th/9602114] [inSPIRE].

[8] D.R. Morrison and C. Vafa, Compactifications of F-theory on Calabi-Yau threefolds. 2, Nucl. Phys. B 476 (1996) 437 [hep-th/9603161] [INSPIRE].

[9] A. Sen, F theory and orientifolds, Nucl. Phys. B 475 (1996) 562 [hep-th/9605150] [INSPIRE].

[10] R. Friedman, J. Morgan and E. Witten, Vector bundles and F-theory, Commun. Math. Phys. 187 (1997) 679 [hep-th/9701162] [INSPIRE].

[11] P.S. Aspinwall and D.R. Morrison, Point-like instantons on K3 orbifolds, Nucl. Phys. B 503 (1997) 533 [hep-th/9705104] [INSPIRE].

[12] L.B. Anderson, J.J. Heckman and S. Katz, T-branes and geometry, JHEP 05 (2014) 080 [arXiv:1310.1931] [INSPIRE].

[13] A.P. Braun, Y. Kimura and T. Watari, The Noether-Lefschetz problem and gauge-group-resolved landscapes: F-theory on K3 $\times$ K3 as a test case, JHEP 04 (2014) 050 [arXiv: 1401.5908] [INSPIRE].

[14] N. Cabo Bizet, A. Klemm and D. Vieira Lopes, Landscaping with fluxes and the E 8 Yukawa point in F-theory, arXiv: 1404.7645 [INSPIRE].

[15] M. Cvetič, A. Grassi, D. Klevers, M. Poretschkin and P. Song, Origin of abelian gauge symmetries in heterotic/F-theory duality, JHEP 04 (2016) 041 [arXiv:1511.08208] [INSPIRE].

[16] S. Mizoguchi and T. Tani, Looijenga's weighted projective space, Tate's algorithm and Mordell-Weil Lattice in F-theory and heterotic string theory, JHEP 11 (2016) 053 [arXiv: 1607.07280] [INSPIRE].

[17] F.R. Cossec and I.V. Dolgachev, Enriques surfaces I, Progress in Mathematics volume 76, Birkhäuser, Germany (1989).

[18] R. Miranda and U. Persson, On extremal rational elliptic surfaces, Math. Z. 193 (1986) 537.

[19] I. Naruki, Configurations related to maximal rational elliptic surfaces, Adv. Stud. Pure Math. 8 (1986) 315.

[20] M. Schütt and T. Shioda, Elliptic surfaces, Adv. Studies Pure Math. 60 (2010) 51 [arXiv: 0907.0298].

[21] G.W. Moore, Les Houches lectures on strings and arithmetic, hep-th/0401049 [INSPIRE].

[22] T. Shioda, K3 surfaces and sphere packings, J. Math. Soc. Japan 60 (2008) 1083.

[23] Y. Kimura, Gauge symmetries and matter fields in F-theory models without sectioncompactifications on double cover and Fermat quartic K3 constructions times K3, arXiv: 1603.03212 [INSPIRE]. 
[24] Y. Kimura, Gauge groups and matter fields on some models of F-theory without section, JHEP 03 (2016) 042 [arXiv: 1511.06912] [INSPIRE].

[25] I. Shimada and D.-Q. Zhang, Classification of extremal elliptic K3 surfaces and fundamental groups of open K3 surfaces, Nagoya Math. J. 161 (2001) 23 [math/0007171].

[26] T. Shioda and H. Inose, On singular K3 surfaces, in Complex analysis and algebraic geometry, in W.L. Jr. Baily and T. Shioda eds., Iwanami Shoten, Tokyo Japan (1977).

[27] M. Bershadsky et al., Geometric singularities and enhanced gauge symmetries, Nucl. Phys. B 481 (1996) 215 [hep-th/9605200] [inSPIRE].

[28] M. Schütt, Elliptic fibrations of some extremal K3 surfaces, Rocky Mount. J. Math. 37 (2007) 609 [math/0412049].

[29] K. Kodaira, On compact analytic surfaces III, Ann. Math. 78 (1963) 1.

[30] K. Becker and M. Becker, M theory on eight manifolds, Nucl. Phys. B 477 (1996) 155 [hep-th/9605053] [INSPIRE].

[31] S. Sethi, C. Vafa and E. Witten, Constraints on low dimensional string compactifications, Nucl. Phys. B 480 (1996) 213 [hep-th/9606122] [INSPIRE].

[32] E. Witten, On flux quantization in M-theory and the effective action, J. Geom. Phys. 22 (1997) 1 [hep-th/9609122] [INSPIRE].

[33] S. Gukov, C. Vafa and E. Witten, CFT's from Calabi-Yau four folds, Nucl. Phys. B 584 (2000) 69 [Erratum ibid. B 608 (2001) 477] [hep-th/9906070] [INSPIRE].

[34] K. Dasgupta, G. Rajesh and S. Sethi, M theory, orientifolds and G-flux, JHEP 08 (1999) 023 [hep-th/9908088] [INSPIRE].

[35] P.S. Aspinwall and R. Kallosh, Fixing all moduli for M-theory on K3 $\times$ K3, JHEP 10 (2005) 001 [hep-th/0506014] [INSPIRE].

[36] I.I. Piatetski-Shapiro and I.R. Shafarevich, A Torelli theorem for algebraic surfaces of type K3, Izv. Akad. Nauk SSSR Ser. Mat. 35 (1971) 530.

[37] D.R. Morrison and W. Taylor, Sections, multisections and U(1) fields in F-theory, arXiv: 1404.1527 [INSPIRE].

[38] V. Braun and D.R. Morrison, F-theory on genus-one fibrations, JHEP 08 (2014) 132 [arXiv: 1401.7844] [INSPIRE].

[39] L.B. Anderson, I. García-Etxebarria, T.W. Grimm and J. Keitel, Physics of F-theory compactifications without section, JHEP 12 (2014) 156 [arXiv:1406.5180] [INSPIRE].

[40] D. Klevers et al., F-Theory on all toric hypersurface fibrations and its Higgs branches, JHEP 01 (2015) 142 [arXiv: 1408.4808] [inSPIRE].

[41] I. García-Etxebarria, T.W. Grimm and J. Keitel, Yukawas and discrete symmetries in F-theory compactifications without section, JHEP 11 (2014) 125 [arXiv:1408.6448] [INSPIRE].

[42] C. Mayrhofer, E. Palti, O. Till and T. Weigand, Discrete gauge symmetries by Higgsing in four-dimensional F-theory compactifications, JHEP 12 (2014) 068 [arXiv:1408.6831] [INSPIRE].

[43] C. Mayrhofer, E. Palti, O. Till and T. Weigand, On discrete symmetries and torsion homology in F-theory, JHEP 06 (2015) 029 [arXiv: 1410.7814] [INSPIRE].

[44] V. Braun, T.W. Grimm and J. Keitel, Complete intersection fibers in F-theory, JHEP 03 (2015) 125 [arXiv:1411.2615] [INSPIRE]. 
[45] M. Cvetič et al., F-theory vacua with $\mathbb{Z}_{3}$ gauge symmetry, Nucl. Phys. B 898 (2015) 736 [arXiv: 1502.06953] [INSPIRE].

[46] L. Lin, C. Mayrhofer, O. Till and T. Weigand, Fluxes in F-theory compactifications on genus-one fibrations, JHEP 01 (2016) 098 [arXiv: 1508.00162] [INSPIRE].

[47] Y. Kimura, Gauge groups and matter spectra in F-theory compactifications on genus-one fibered Calabi-Yau 4-folds without section - Hypersurface and double cover constructions, arXiv: 1607.02978 [INSPIRE].

[48] Y. Kimura, Discrete gauge groups in F-theory models on genus-one fibered Calabi-Yau 4-folds without section, JHEP 04 (2017) 168 [arXiv: 1608.07219] [INSPIRE].

[49] S. Kondo, Automorphisms of algebraic K3 surfaces which act trivially on Picard groups, J. Math. Soc. Japan 44 (1992) 75.

[50] S. Kondo, Type II degenerations of K3 surfaces, Nagoya Math J. 99 (1985) 11.

[51] V.V. Nikulin, Integral symmetric bilinear forms and some of their applications, Math. USSR Izv. 14 (1980) 103.

[52] J. Milnor, On simply connected 4-manifolds, talk given at the Symposium Internacional de Topologia Algebraica (International Symposium on Algebraic Topology), Mexico City, Mexico (1958).

[53] T. Shioda, On the Mordell-Weil lattices, Comment. Math. Univ. St. Pauli 39 (1990) 211.

[54] K. Oguiso and T. Shioda, The Mordell-Weil lattice of a rational elliptic surface, Comment. Math. Univ. St. Pauli 40 (1991) 83.

[55] I. Shimada, On elliptic K3 surfaces, Michigan Math. J. 47 (2000) 423 [math/0505140]. 